\title{
Deep Learning Applied to the Detection of Masks on Faces
}

\author{
Jose Luis Calderon Osorno, Edmundo Rene Duran Camarillo, \\ and Silvestre Ascencion García Sanchez
}

\begin{abstract}
Derived from the pandemic COVID19 that we are currently experiencing as a security measure it is very important that people wear face masks especially in public places, to try to minimize the spread of the SARS-Cov2 virus; this Project consisted in the detection of faces with and without face masks applying Deep learning neural networks, it was developed by using Python and the libraries TensorFlow and OpenCV which allowed to apply learning rules to artificial vision systems. The above would allow the installation of artificial vision systems in public places soon to warn and invite people to wear face masks when detected by the system.
\end{abstract}

Keywords - artificial vision, COVID-19, Deep Learning, TensorFlow.

\section{INTRODUCTION}

During the present year 2020 the most important event that has occurred worldwide there is the arising of the COVID19 pandemic, in the far East in the People's Republic of China in the Wuhan City, Hubei province. Such event shook the world, paralyzing the global economy, making humanity to reevaluate their priorities. The SARS-Cov2 virus has claimed more than 761779 lives worldwide, with several 21.2 million 21294845 confirmed infected cases, unfortunately in our country the health crisis has reported 525733 deaths with more than 57023 infected people according to the World Health Organization.

Deep learning is a type of automatic learning in which a model learns to perform classification tasks directly from images, text, or sound. Deep learning is generally implemented using a neural network architecture. The term "Deep" refers to the number of layers of the network: The more layers, the deeper the network is. Traditional neural networks contain only 2 or 3 layers, while deep networks can have hundreds. Deep learning is especially suitable for identification applications such as facial recognition, text translation, voice recognition and advanced driver assistance systems, including lane classification and traffic sign recognition.

A Deep neural network combines multiple layers of nonlinear processing, using simple elements that operate in parallel and inspired by biological nervous systems. It consists of an input layer, several hidden layers, and an output layer. Layers are interconnected through nodes or neurons, and each layer uses the output of the previous layer as input.

Submitted on September 18, 2021

Published on October 09, 2021.

Jose Luis Calderon Osorno, Instituto Politecnico Nacional, UPIIH, San

Agustin Tlaxiaca, Mexico.

(e-mail: jcalderono@ipn.mx).
The training of a Deep learning model can take hours, days or weeks depending on the size of the data and the amount of processing power you have available. The selection of a computing resource is a critical consideration when configurating your workflow.

The present Project proposes to apply the Deep learning paradigm in artificial neural networks derived from artificial intelligence using Python, TensorFlow and OpenCV libraries to perform the detection of people with and without masks, this would allow the development and installation of artificial vision systems in public places in a near future, to warn and invite people to wear the mask when detected by the system. This article is derivative of the SIP 20201970 Project

\section{DEVELOPMENT}

This stem was developed with the help of the open-source platform Anaconda 3, which was used to execute the performed codes that were based on Python, for which it was required to process large volumes of information, as well as to apply automatic learning.

The Anaconda package was used as the software that contributed to the data processing, a virtual environment was built, which had the function of storing all the libraries that were used in this Project, it was created through a command that allowed us to create a library which we called "MASK DETECTOR" (Fig. 1), running on the "Anaconda prompt" program, that was based on 3.7.7 Python version through the Anaconda 3 platform.

Documents $\backslash$ UPIIH $>$ conda create $-n$ DETECTOR_CUBREBOCAS python=3.7.7 anaconda

Fig. 1. Executed command in "Anaconda prompt" program.

Then, it was necessary to install the libraries required for the execution of the project, these are the ones listed below:

1. TensorFlow.

2. Numpy.

3. Imutils.

4. Open CV.

A search and compilation were made in databases with around 700 images of people's faces with and without face masks, which served as basis for the training of the multilayer neural network deep learning. A sample of the set of images is observed in Fig. 2 and Fig. 2 b.

It was developed a program coded in python for the

Edmundo Rene Duran Camarillo, Instituto Politecnico Nacional, ESCOM, Mexico.

(e-mail: eduranc@ipn.mx).

Silvestre Ascencion Garcia Sanchez, Instituto Politecnico Nacional, UPIIH, San Agustinc Tlaxiaca, Mexico.

(e-mail: sagarcias@ipn.mx). 
detection of faces with and without masks, for its execution process, it was first necessary to load the libraries for its operation (Fig. 3). In the same way, a code segment was drafted and written, where the detection cycle was made for the identification of faces and finally it was selected, where the prediction of the use of face masks was made, only if a face was detected. (Fig. 4).

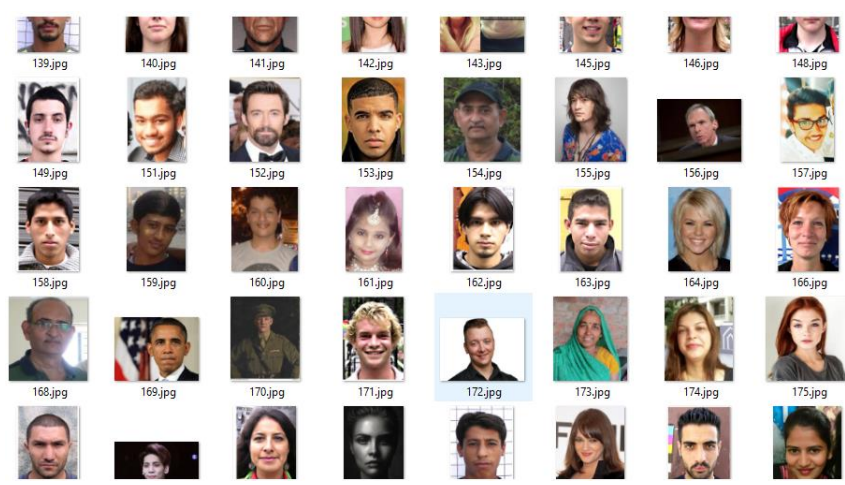

Fig. 2 a. Sample of images of people without face masks.

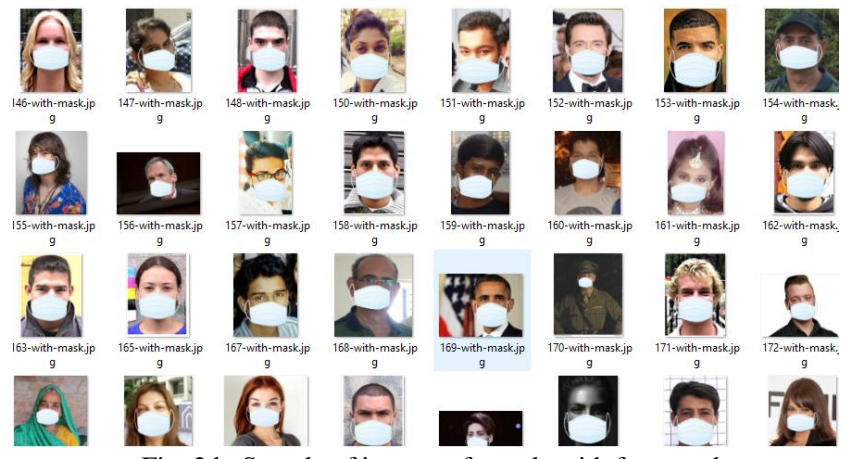

Fig. 2 b. Sample of images of people with face masks.

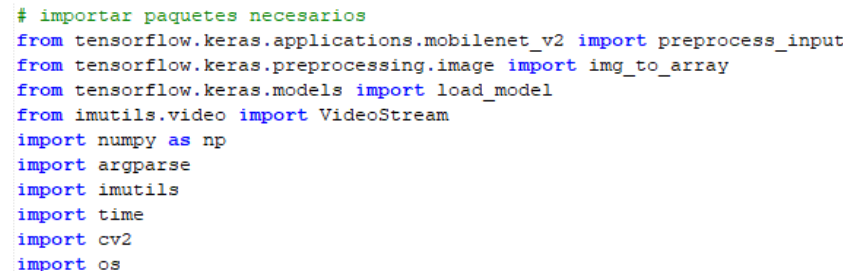

Fig. 3. Libraries Load.

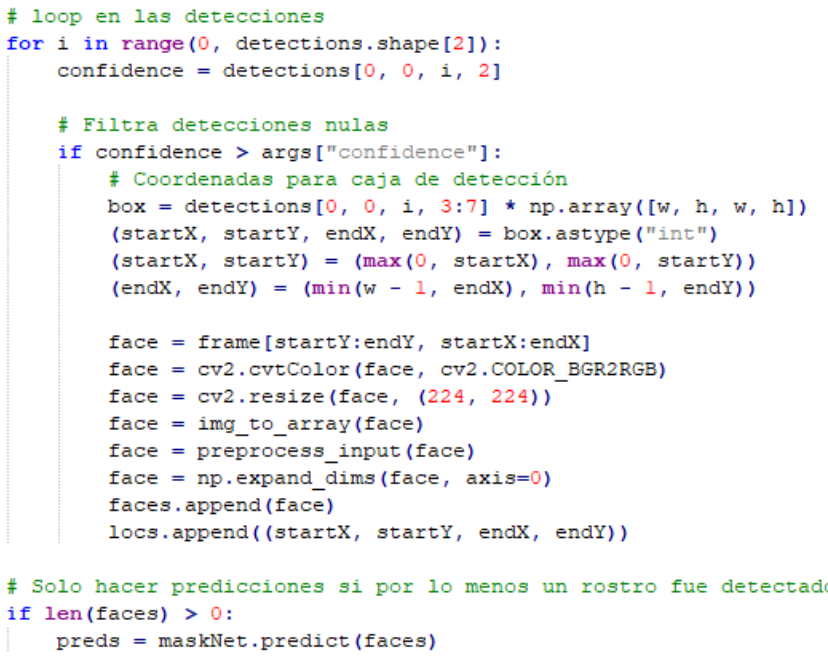

Fig. 4. Code for face detection.

A subroutine was written to print the legend "Loading model ...", which aims to indicate if the mask detection model is being loaded and later it was started the video through the connected webcam defined as "src", the value of this variable was used to indicate which web camera would be used (Fig. $5)$, the number 0 is the default camera.

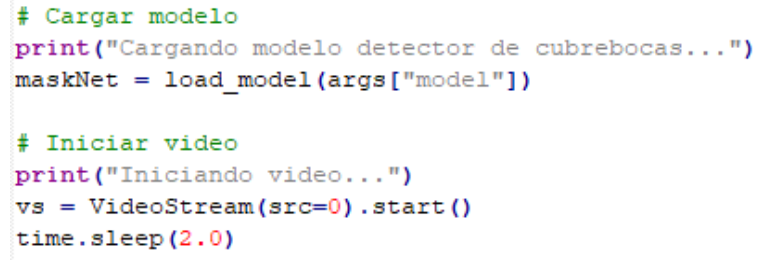

Fig. 5. Model loading and Starting video.

Subsequently it was determined through the models created with the images that served as a training set, if there was a face with or without a mask, once this was done a label was programmed on the frame created for the face, which would indicate if a face with or without mask was detected, in the case of having a face mask the frame and the legend would be indicated with a green label, otherwise since there were no face masks, the frame and the legend would be indicated with a red label, finally, a window was created where the video was shown and in the case of wanting to leave form this window you must press the letter "q" (Fig. 6). The execution of the program is carried out with the Anaconda command prompt (Fig. 7).

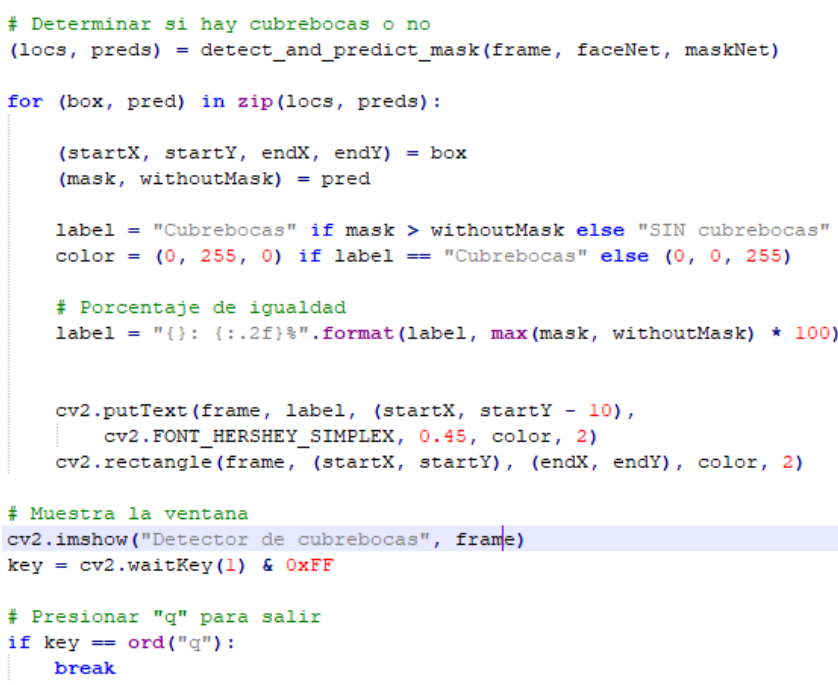

Fig. 6. Show indication of the use of face masks.

\section{python Detector_de_cubrebocas.py}

Fig. 7. Command to run the developed program.

\section{RESUlts}

To validate the efficiency of the prototype of the developed system many tests were carried out, executing the code through Anaconda prompt, putting this in operation (fig.8), in the same way that it was tested with different people and different types of face masks, having a very efficient and satisfactory system behavior. (Fig. 9 a and Fig. 9 b).

Fig. 8. Legend when running the program. 


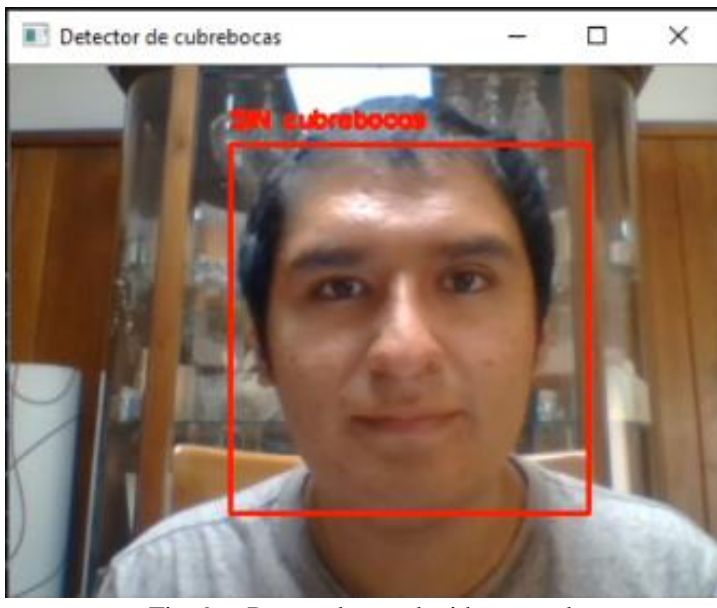

Fig. 9 a. Person detected without mask.

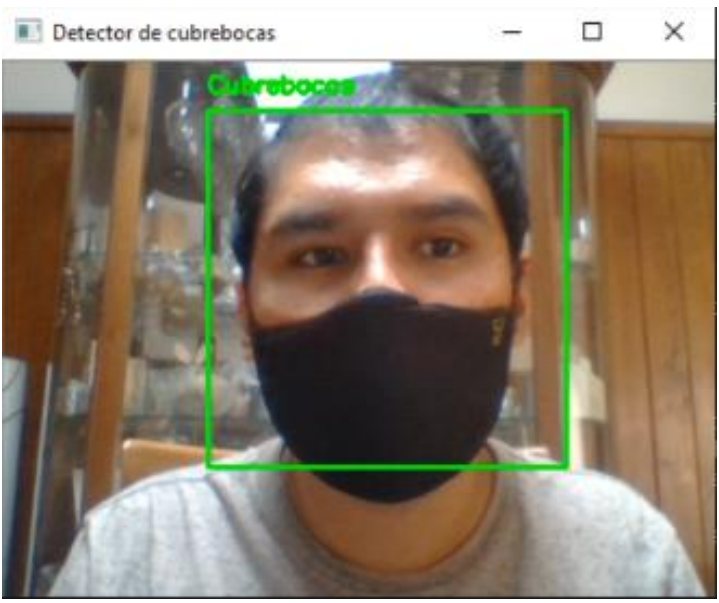

Fig. 9 b. Person detected with mask.

Finally, the system was trained with a database of approximately 700 faces, in 6 seconds time, after performing multiple tests, the performance of the system was very satisfactory, with a high degree of efficiency in detecting a face of a person wearing a mask, preferably light color, the detection of the face with or without mask was carried out in 6 seconds. However, some problems were found when wearing dark-colored masks, especially when detecting in places with poor lighting, the recognition came to fail on some occasions.

\section{CONCLUSIONS}

A prototype of a system was developed for detecting the faces of people with and without masks using deep learning, based on open-source tools, the Anaconda platform 3, code developed in Python and the TensorFlow libraries, Numpy, Imutils, and OpenCV. The prototype showed a high degree of efficiency in detection when the person wore light colored masks.

\section{REFERENCES}

[1] I. Goodfellow, Y. Bengio and A. Courville, Deep learning, MIT press, 2016.

[2] D. Graupe, Deep Learning Neural Networks. World Scientific Publishing, 2016

[3] C.C. Arggarwal, "Neural Networks and Deep Learning", Springer International Publishing, vol.10, pp. 978-3, 2018.
[4] A. Géron, Hands-on machine learning with Scikit-Learn, Keras, and TensorFlow: Concepts, tools, and techniques to build intelligent systems, O'Reilly Media, 2019.

[5] M. Dawson, Python Programming, 3rd. ed. Course Technology Cengage Learning, 2010.

[6] https://www.anaconda.com/products/individual.

[7] https://docs.opencv.org/master/d6/d00/tutorial_py_root.html.

[8] https://www.toptal.com/machine-learning/de-resolver-ecuaciones-aaprendizaje-profundo-un-tutorial-de-tensorflow-python

[9] https://www.luisllamas.es/machine-learning-con-tensorflow-y-kerasen-python/.

[10] https://omes-va.com/reconocimiento-facial-python-opencv/.

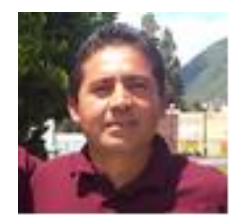

Jose Luis Calderon Osorno obtained the title of electronics engineer from Instituto Tecnologico de Orizaba in Orizaba, Mexico in 1991. He obtained a Master of Science degree from the CINVESTAV in the Instituto Politecnico Nacional in Mexico City, Mexico in 1995.

He was a professor of the Escuela Superior de Computo in the Instituto Politecnico Nacional from 1995 to 2015. Currently, he is a full professor in the Unidad Profesional Interdisciplinaria de Ingenieria Campus Hidalgo, Instituto Politecnico Nacional in San Agustin Tlaxiaca Mexico. His areas of interest are artificial intelligence, neural networks, genetic algorithms, expert systems, fuzzy control systems, artificial vision, pattern recognition and robotics.

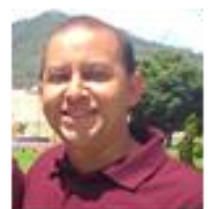

Edmundo Rene Duran Camarillo obtained the title of Electronics Engineer from Instituto Tecnologico de Orizaba in Orizaba, Mexico in 1993. He obtained the Master of Science degree from the CINVESTAV, Instituto Politecnico Nacional in Mexico City, Mexico in 1994

$\mathrm{He}$ is a senior research professor at the Escuela Superior de Computo, Instituto Politecnico Nacional in Mexico City, Mexico. His areas of interest are automation and control, artificial intelligence, neuro-fuzzy systems, artificial neural networks, and expert systems.

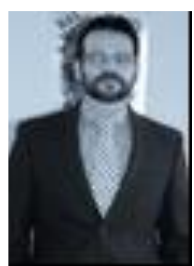

Silvestre Ascencion Garcia Sanchez was born in Madero city, Mexico on 11 July of 1975 . He obtained the title of physics from Universidad Nacional Autonoma de Mexico in Mexico City, Mexico in 2003. He obtained a Master of Science degree from the Escuela Superior de Ingenieria Mecanica y Electrica, Campus Culhuacan, Instituto Politecnico Nacional in Mexico City, Mexico in 2003.

He was full professor at ESIME CULHUACAN, Instituto Politecnico Nacional from 1999-2016 and asocciate graduate professor at SEPI ESIME CULHUACAN, Instituto Politecnico Nacional from 2005-2008. $\mathrm{He}$ is a full professor in the Unidad Profesional Interdisciplinaria de Ingenieria Campus Hidalgo, Instituto Politecnico Nacional in San Agustin Tlaxiaca Mexico. His areas of interest are artificial intelligence, neural networks, genetic algorithms, data mining and adaptive filters. 